\title{
Impacto de la espectrometría de masas por MALDI-TOF MS en la identificación rápida de bacterias aeróbicas y anaeróbicas de importancia clínica
}

\author{
Paulette Legarraga, Marcela Moraga, Marusella Lam, Enrique Geoffroy, Cecilia Zumarán y Patricia García
}

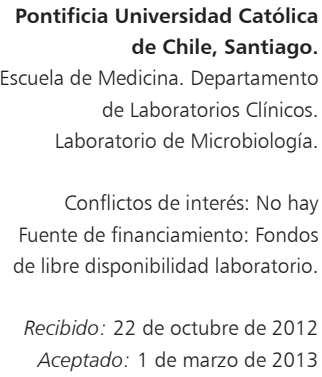

Pontificia Universidad Católica de Chile, Santiago. Escuela de Medicina. Departamento de Laboratorios Clínicos Laboratorio de Microbiología.

Conflictos de interés: No hay Fuente de financiamiento: Fondos de libre disponibilidad laboratorio.

Recibido: 22 de octubre de 2012 Aceptado: 1 de marzo de 2013

Correspondencia a: Patricia García Cañete pgarcia@med.puc.c

\section{Impact of mass spectrometry by MALDI-TOF MS for the rapid identification of aerobic and anaerobic bacteria of clinical importance}

Background: MALDI-TOF MS (Matrix Assisted Laser Desorption Ionization -Time of Flight Mass Spectrometry) technology, recently introduced in the microbiology laboratory has proven to be a precise and rapid method for bacterial identification. Objective: To evaluate the performance, costs associated and turnaround time of MALDI-TOF in a routine laboratory. Material and Method: Five hundred and sixty one clinical isolates (281 aerobes and 280 anaerobes) previously identified by conventional methods were evaluated. Discordances were resolved by means of $16 \mathrm{~S}$ rRNA sequencing. Results: MALDI-TOF identified $95,7 \%$ of the aerobes isolates and $86,4 \%$ of the anaerobes. The groups with better performance were the enterobacteriacea and Bacteroides spp with $95 \%$ and $100 \%$ identification at the species level. The error rate of MALDI-TOF and conventional methods compared to sequencing was $0,39 \%$ and $9,4 \%$ respectively. The costs associated were 8 times lower with a turnaround time of 6 hours. Conclusion: MALDI-TOF proved to be simple, precise and less expensive technology compared to the traditional methods.

Key words: MALDI-TOF MS, mass spectrometry, identification.

Palabras clave: MALDI-TOF MS, espectrometría de masas, identificación.

\section{Introducción}

L a identificación de microorganismos en el laboratorio de microbiología se ha llevado a cabo tradicionalmente mediante pruebas fenotípicas como son la caracterización macroscópica de las colonias, características tintoriales y pruebas bioquímicas. Un avance importante fue la automatización de estas pruebas permitiendo un diagnóstico más rápido. Sin embargo, a pesar de los avances, estas tecnologías aún requieren de tiempos de incubación que pueden tomar varias horas y con un alto costo asociado. Por otro lado, la espectrometría de masas, utilizada ampliamente en el campo de la investigación, a pesar de su alta especificidad, ha tenido un rol menor en el laboratorio clínico.

El desarrollo de la tecnología de MALDI-TOF MS (matrix assisted laser desorption ionization-time of flight-mass spectrometry, en español: desorción láser asistida por la matriz con detección de masas por tiempo de vuelo MALDI-TOF MS) hace más de 30 años ha permitido la utilización de la espectrometría de masas en el diagnóstico microbiológico de rutina. Permite la identificación de microorganismos mediante el análisis de proteínas, principalmente ribosomales, a partir de colonias o directamente de muestras a través de la creación de un espectro de masas el que es específico para cada especie ${ }^{1,2}$. La implementación de MALDI-TOF MS es sencilla, necesitando sólo un par de días para la capacitación del personal, la identificación es rápida y el costo por cada muestra es inferior al costo de otros métodos diagnósticos.

El número de publicaciones con respecto al desempeño de MALDI-TOF MS en la identificación de microorganismos ha ido aumentando de manera importante. Esta tecnología ha demostrado ser confiable, específica y rápida para la identificación de distintas bacterias aeróbicas y anaeróbicas. Los estudios abarcan bacterias de distintas familias como: Enterobacteriaceae, bacilos gramnegativos no fermentadores, cocáceas grampositivas, levaduras y micobacterias ${ }^{3,4}$. Si bien este método no ha sido aún aprobado por la FDA en los Estados Unidos de América, ha sido utilizado ampliamente en laboratorios clínicos europeos. En Chile no hay publicaciones que evalúen su rendimiento.

El objetivo de este trabajo fue evaluar la utilidad y el desempeño de MALDI-TOF MS en la identificación de bacterias aeróbicas y anaeróbicas de importancia clínica en un laboratorio de rutina. Además se compararon los costos y el tiempo necesario para cada identificación en comparación a los métodos utilizados hasta ese momento de manera rutinaria en el laboratorio. 


\section{Material y Método}

Aislados bacterianos. Entre abril y agosto de 2011, en el Laboratorio de Microbiología de la Pontificia Universidad Católica de Chile (PUC) se analizaron 561 aislados bacterianos provenientes de muestras clínicas de pacientes (281 aislados aeróbicos y 280 anaeróbicos estrictos) identificados previamente por métodos convencionales y que estaban almacenadas en un cepario. La selección de las cepas fue realizada considerando aquellos aislados menos frecuentes y con menor información en la literatura. Estos se distribuyeron en 105 Enterobacteriaceae (11 géneros), 29 bacilos gramnegativos no fermentadores (6 géneros), 74 fastidiosos ( 9 géneros), 64 cocáceas positivas (13 géneros), 9 bacilos grampositivos ( 5 géneros) y 280 anaerobios estrictos ( 8 géneros). El detalle de los aislados estudiados se muestra en la Tabla 1. Todos fueron identificados por MALDI-TOF MS. El resultado obtenido fue comparado con la identificación por métodos convencionales. Las discordancias fueron resueltas por secuenciación del ARNr 16S.

Del total de los aislados estudiados, los correspondientes al grupo de los fastidiosos se encontraban congelados a $-80^{\circ} \mathrm{C}$ en caldo $\mathrm{BHI}$ y glicerol al $15 \%$ con suero de caballo. Los aislados fueron sembrados en agar Polivitex bioMerieux ${ }^{\circledR}$ e incubados durante $48-72$ h a $35^{\circ} \mathrm{C}$ y $5 \%$ $\mathrm{CO}_{2}$. Enterobacteriaceae, bacilos gramnegativos no fermentadores y cocáceas grampositivas se encontraban congelados en caldo BHI con glicerol al $15 \%$ a $-20^{\circ} \mathrm{C}$. Los subcultivos se realizaron en placas de agar soya tripticasa bioMerieux ${ }^{\circledR}$ e incubados durante $24 \mathrm{~h}$ a $35^{\circ} \mathrm{C}$ y $5 \% \mathrm{CO}_{2}$. Los aislados anaerobios estrictos se encontraban a $-80^{\circ} \mathrm{C}$ en sangre de cordero y fueron subcultivados en placas agar sangre anaerobio en jarra con anaerogen durante $72 \mathrm{~h}$ a $35^{\circ} \mathrm{C}$.

Todos los aislados fueron subcultivados dos veces consecutivas antes de su estudio.

\section{Espectrometría de masas (MALDI-TOF MS)}

Un inoculo tomado a partir de una colonia aislada en cultivo puro fue depositado por duplicado sobre una tarjeta de análisis (Bruker Daltonics, Bremen, Alemania) y se dejó secar a temperatura ambiente. Luego, los pocillos fueron cubiertos con $1 \mu \mathrm{L}$ de matriz (solución saturada de ácido $\alpha$-ciano-4-hidroxicinámico [HCCA; Bruker Daltonics] en 50\% acetonitrilo y 2,5\% ácido trifluoroacético). MALDI-TOF MS fue realizado en el equipo Microflex LT utilizando el software FlexControl (versión 3.0 Bruker Daltonics). Los parámetros del equipo fueron ajustados según recomendaciones del fabricante. Para la calibración del espectrómetro se utilizó una prueba estándar consistente en el perfil proteico de una cepa de Escherichia coli DH5 péptido alfa que posee proteínas adicionales (BTS; Bruker Daltonics). El estándar BTS se utilizó también como control positivo para validar la corrida. Los espectros para cada aislado fueron obtenidos con 240 disparos del láser en seis regiones distintas en el mismo pocillo y dichos espectros fueron analizados por el software MALDI Biotyper RTC 3.0 (Bruker Daltonics, Bremen, Alemania). Los espectros son creados considerando el tiempo requerido por las proteínas para llegar al detector lo que depende de la relación masa/carga de éstas. Cada espectro al ser comparado con la base de datos permite la asignación de un puntaje. Los criterios de iden-

Tabla 1. Número de aislados estudiados por grupo bacteriano y detalle de los géneros estudiados

\begin{tabular}{|c|c|c|}
\hline Aislados estudiados & $\mathrm{n}$ aislados & Género estudiados ( número de aislados) \\
\hline $\begin{array}{l}\text { Bacilos gramnegativos, Enterobacteriaceae } \\
\text { (11 géneros) y Aeromonas spp }\end{array}$ & 105 & $\begin{array}{l}\text { Escherichia coli (27), Enterobacter spp (21), Klebsiella pneumoniae (11), Klebsiella oxytoca (5), Morganella spp (7), } \\
\text { Proteus spp (6), Hafnia alvei (8), Pantoea agglomerans (5), Providencia (1), Citrobacter spp (4), Salmonella spp (2), } \\
\text { Serratia spp (2), Aeromonas (6) }\end{array}$ \\
\hline $\begin{array}{l}\text { Bacilos gramnegativos no fermentadores } \\
\text { ( } 6 \text { géneros) }\end{array}$ & 29 & $\begin{array}{l}\text { Achromobacter spp (4), grupo Acinetobacter baumannii (3), Pseudomonas spp (12), Ralstonia spp (3), Sphingo- } \\
\text { bacterium multivorum (1), Stenotrophomonas spp (6) }\end{array}$ \\
\hline Fastidiosos (9 géneros) & 74 & $\begin{array}{l}\text { Haemophilus spp (25), Eikenella corrodens (13), Kingella kingae (5), Capnocytophaga spp (12), Moraxella catarrhalis } \\
\text { (11), Aggregatibacter spp (4), Bordetella brochiseptica (2), Actinobacillus suis (1), Pasteurella multocida (1) }\end{array}$ \\
\hline $\begin{array}{l}\text { Cocáceas grampositivas habituales } \\
\text { (4 géneros ) }\end{array}$ & 41 & Staphylococcus aureus (12), Staphylococcus coagulasa negativa (18), Streptococcus spp (7), Enterococcus spp (4) \\
\hline $\begin{array}{l}\text { Cocáceas grampositivas inhabituales } \\
\text { (9 géneros ) }\end{array}$ & 23 & $\begin{array}{l}\text { Abiotrophia spp (3), Aerococcus spp (4), Granulicatella spp (3), Kokuria spp (2), Micrococcus spp (2), Rothia muci- } \\
\text { laginosa (3), Gemella spp (1), Lactococcus spp (2), Leuconostoc spp (3) }\end{array}$ \\
\hline Bacilos grampositivos (5 géneros) & 9 & $\begin{array}{l}\text { Lactobacillus spp (4), Rhodococcus spp (2), Microbacterium hydrocarbonoxydans (1), Brevibacterium spp (1), } \\
\text { Corynebacterium aurimucosum (1) }\end{array}$ \\
\hline Anaerobios estrictos (8 géneros) & 280 & $\begin{array}{l}\text { Propionibacterium spp (91), Bacteriodes spp (87), Clostridium spp (58), Prevotella spp (40), Peptostreptococcus } \\
\text { anaerobius (1), Finegoldia magna (1), Actinobaculum schaalii (1), Slackia exigua (1) }\end{array}$ \\
\hline Total & 561 & \\
\hline
\end{tabular}


tificación señalados por el fabricante son los siguientes: puntaje $>2,000$ indica identificación a nivel de especie, puntaje entre 1,700-1,999 indica identificación confiable sólo a nivel de género, mientras que valores $<1,700$ no permiten identificación ${ }^{5}$.

\section{Identificación microbiológica convencional}

La identificación convencional de bacterias aeróbicas se realizó por métodos automatizados (tarjeta de identificación VITEK GN+® en VITEK ${ }^{\circledR} 2$, bioMérieux) y por sistemas manuales comerciales API NH® (bioMérieux), API NE® (bioMérieux), API STREPTO ID32® (bioMérieux) y otras pruebas. En el caso de los anaerobios estrictos, éstos se identificaron mediante tarjeta VITEK ${ }^{\circledR}$ ANC también leída en sistema VITEK ${ }^{\circledR} 2$. (bioMérieux). Estos métodos fueron utilizados según indicaciones del fabricante y según los procedimientos descritos en el laboratorio de microbiología de la PUC.

Resolución de discordancias mediante secuenciación

La extracción del ADN genómico bacteriano se realizó a partir de un cultivo puro, utilizando el kit QIAamp DNA Mini ${ }^{\circledR}$ (Qiagen). Para todas las bacterias se amplificó una región de $1.380 \mathrm{pb}$ del gen que codifica para el ARNr 16S,

Tabla 2. Resultados de la identificación de bacterias aerobias según grupo de microorganismo, número y porcentaje de aislados identificados a nivel de género y especie y número de discordancias observadas

\begin{tabular}{|c|c|c|c|c|}
\hline \multirow{2}{*}{$\begin{array}{l}\text { Microorganismos } \\
\text { ( } n^{\circ} \text { de aislados) }\end{array}$} & \multicolumn{2}{|c|}{ Identificación adecuada } & \multicolumn{2}{|c|}{ Identificación inadecuada } \\
\hline & $\begin{array}{l}\% \text { aislados } \\
\text { identificados } \\
\text { a nivel de } \\
\text { género }\end{array}$ & $\begin{array}{c}\% \text { aislados } \\
\text { identificados } \\
\text { a nivel de } \\
\text { género y } \\
\text { especie }\end{array}$ & $\begin{array}{c}\% \text { de no } \\
\text { identificadas* }\end{array}$ & $\begin{array}{c}\% \text { de } \\
\text { discordancias** }\end{array}$ \\
\hline $\begin{array}{l}\text { Enterobacteriaceae y otros } \\
\text { bacilos gram negativos (105) }\end{array}$ & $\begin{array}{c}4 \% \\
(4 / 105)\end{array}$ & $\begin{array}{c}95 \% \\
(100 / 105)\end{array}$ & $\begin{array}{c}1 \% \\
(1 / 105)\end{array}$ & $0 / 100$ \\
\hline $\begin{array}{l}\text { Bacilos gramnegativos no } \\
\text { fermentadores (29) }\end{array}$ & $\begin{array}{l}3,5 \% \\
(1 / 29)\end{array}$ & $\begin{array}{c}93 \% \\
(27 / 29)\end{array}$ & $\begin{array}{l}3,5 \% \\
(1 / 29)\end{array}$ & $0 / 29$ \\
\hline Fastidiosos (74) & $\begin{array}{c}8 \% \\
(6 / 74)\end{array}$ & $\begin{array}{c}87 \% \\
(64 / 74)\end{array}$ & $\begin{array}{c}4 \% \\
(3 / 74)\end{array}$ & $\begin{array}{c}1 \% \\
(1 / 74)\end{array}$ \\
\hline $\begin{array}{l}\text { Cocáceas grampositivas } \\
\text { habituales (41) }\end{array}$ & $\begin{array}{l}15 \% \\
(6 / 41)\end{array}$ & $\begin{array}{c}85 \% \\
(35 / 41)\end{array}$ & $0 / 41$ & $0 / 41$ \\
\hline $\begin{array}{l}\text { Cocáceas grampositivas } \\
\text { inhabituales (23) }\end{array}$ & $\begin{array}{l}22 \% \\
(5 / 23)\end{array}$ & $\begin{array}{c}61 \% \\
(14 / 23)\end{array}$ & $\begin{array}{l}17 \% \\
(4 / 23)\end{array}$ & $0 / 23$ \\
\hline Bacilos grampositivos (9) & $\begin{array}{l}22 \% \\
(2 / 9)\end{array}$ & $\begin{array}{l}56 \% \\
(5 / 9)\end{array}$ & $\begin{array}{l}22 \% \\
(2 / 9)\end{array}$ & $0 / 9$ \\
\hline \multirow[t]{2}{*}{ Total (281) } & $\begin{array}{c}8,6 \% \\
(24 / 281)\end{array}$ & $\begin{array}{c}87,1 \% \\
(245 / 281)\end{array}$ & $\begin{array}{c}3,9 \% \\
(11 / 281)\end{array}$ & $\begin{array}{c}0,4 \% \\
(1 / 281)\end{array}$ \\
\hline & \multicolumn{2}{|c|}{$\begin{array}{c}95,7 \% \\
(269 / 281)\end{array}$} & \multicolumn{2}{|c|}{$\begin{array}{c}4,3 \% \\
(12 / 281)\end{array}$} \\
\hline
\end{tabular}

*No identificadas: Citrobacter freundii, Sphingobacterium multivorum, Haemophilus spp, Capnocytophaga spp, Eikenella spp. **Discordancias: Actinobacillus suis por secuencia, Actinobacillus ureae por MALDI-TOF. utilizando los partidores: 5'-AGT TTG ATC CTG GCT CAG-3'6 y 5'-AGG CCC GGG AAC GTA TTC AC- $3^{7}$. Los productos de RPC fueron visualizados por electroforesis en gel de agarosa al 1,5\% teñido con bromuro de etidio. Los productos amplificados fueron purificados y posteriormente secuenciados en el analizador genético ABI Prism 3130® (Applied Biosystems). Las condiciones de secuenciación han sido descritas previamente ${ }^{8}$. Las secuencias fueron analizadas manualmente y alineadas en $\mathrm{BLAST}^{9}$ y luego comparadas con la información en GenBank ${ }^{10}$. Los resultados fueron interpretados según lo descrito en CLSI MM18- $\mathrm{A}^{11}$.

\section{Resultados}

De los 281 aislados aeróbicos evaluados fue posible obtener una identificación adecuada en 95,7\% (269/281), ya fuese a nivel de género o especie. De estos 269 aislados identificados, 91\% (245/269) fueron identificados correctamente a nivel de especie y el 9\% restante (24/269) fue identificado sólo a nivel de género. En 11 aislados no se obtuvo identificación por MALDI-TOF MS (puntaje $<1.700$ ) correspondiendo a: Citrobacter freundii, Sphingobacterium multivorum, Haemophilus parainfluenzae, Capnocytophaga spp, Eikenella corrodens. Hubo un aislado de Actinobacillus ureae identificado como Actinobacillus suis por MALDI-TOF MS de modo que $4,3 \%$ de los aislados aeróbicos estudiados no fueron identificados adecuadamente (12/281).

En la Tabla 2 se observa el porcentaje de identificación por grupo bacteriano estudiado. El porcentaje más alto de identificación se obtuvo en el grupo de Enterobacteriaceae y otros bacilos gramnegativos. Del total de 105 aislados evaluados, pudo identificarse $95 \%$ a nivel de género y especie, $4 \%$ sólo a nivel de género, no obteniéndose identificación en un aislado de $C$. freundii de los cuatro aislados evaluados. El porcentaje más bajo de identificación se observó en el grupo de bacilos grampositivos, donde, de los nueve aislados evaluados se identificó a $56 \%$ a nivel de género y especie, $22 \%$ sólo a nivel de género y no se obtuvo identificación en el $22 \%$ restante.

De los 280 aislados de bacterias anaerobias estrictas evaluados, 86,4\% (242/280) fueron identificados correctamente. De éstos, 92,5\% (224/242) fueron identificados a nivel de especie y $7,4 \%$ (18/242) sólo a nivel de género. De los 38 aislados que no fueron identificados correctamente, $13,2 \%(37 / 280)$ presentaron puntajes menores a 1,700 . Se observó una discordancia correspondiente a un aislado de Finegoldia magna identificado por MALDITOF MS como Anaerococcus hidrogenalis (Tabla 3). Al examinar los resultados según familias, en el grupo de bacilos gramnegativos, $100 \%$ de los 87 aislados de Bacteroides spp estudiados fueron identificados a nivel de especie, mientras que de los aislados de Prevotella spp 
Tabla 3. Resultados de la identificación de bacterias anaerobias estrictas según grupo de microorganismo, número y porcentaje de aislados identificados a nivel de género y especie y número de discordancias observadas

Microorganismos

(n de aislados evaluados)

Cocáceas Peptostreptococcus

grampositivas

Bacilos

grampositivos

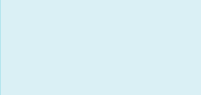

Bacilos

gramnegativos

Bacteroides spp (87)

Prevotella spp (40)

Total (280)

Slackia exigua (2)

Propionibacterium

spp (91)

Clostridium spp (58)
Identificación adecuada

$\%$ identificado a nivel \% identificados a nivel de género

$0 / 2$

Actinobaculum schaalii

$12,5 \%$

$(5 / 40)$

$6,4 \%$

$(18 / 280)$ de género $y$ especie

$50 \%$

$(1 / 2)$

$0 / 2$

$95,6 \%$

$(87 / 91)$

$62 \%$

(36/58)

$100 \%$

$(87 / 87)$

$32,5 \%$

(13/40)

$80 \%$

$(224 / 280)$

Identificación inadecuada

No Discordancias*

identificados

$0 / 2$

$1 / 2$

$0 / 2 \quad 0 / 2$

$0 / 91 \quad 0 / 91$

$26 \%$

$(15 / 58)$

$0 / 87 \quad 0 / 87$

$55 \%$

(22/40)

$13,2 \%$

(37/280)

$86,4 \%$

$13,6 \%$

(242/280)

$(38 / 280)$

*Discordancias: Finegoldia magna por secuencia, Anaerococcus hidrogenalis por MALDI-TOF.

sólo 32,5\% (13/40) fueron identificados a nivel de especie, no obteniéndose identificación en $55 \%$ (22/40). En el caso de Clostridium spp se obtuvo identificación a nivel de especie en $62 \%$ (36/58) no pudiéndose identificar 26\% (15/58). Los aislados de Actinobacullum schalii y Slackia exigua sólo fueron identificados a nivel de género (Tabla 3).

En resumen, de los 561 aislados estudiados se obtuvo identificación adecuada en 511 aislados (91\%), no se obtuvo identificación en 48 aislados (8,5\%) y se observaron 2 discordancias (Tabla 4).

Para el análisis de discordancias sólo se consideró los 513 aislados identificados por MALDI-TOF MS. Las discordancias obtenidas por MALDI-TOF MS correspondieron a un aislado de F. magna identificado como A. hidrogenalis y un aislado de Actinobacillus suis identificado como A. ureae. Al considerar estas dos discordancias, MALDI-TOF MS presenta un porcentaje de error de $0,39 \%$ (2/513). Por otro lado, la identificación convencional presentó un porcentaje de error de 9,4\% (48/513). Hubo identificación errónea por métodos convencionales en 15 aislados aeróbicos, todos ellos a nivel de género (Tabla 5).

En el caso de los aislados anaeróbicos estrictos, hubo discordancias en 33 aislados. A nivel de especie hubo discordancias en 27 aislados de Bacteroides, un aislado de Propionibacterium, dos de Prevotella, mientras que hubo error a nivel de género en un aislado de Slackia, Actinobaculum y Prevotella, respectivamente.
Tabla 4. Resumen número de aislados estudiados, identificaciones adecuadas, no identificados y discordancias encontradas

\begin{tabular}{lcccc|} 
Bacterias & $\begin{array}{c}\mathbf{n} \text { de } \\
\text { aislados }\end{array}$ & $\begin{array}{c}\text { Identificación } \\
\text { adecuada (\%) }\end{array}$ & $\begin{array}{c}\text { Discordancias } \\
\mathbf{( \% )}\end{array}$ & $\begin{array}{c}\text { No identificadas } \\
\text { (\%) }\end{array}$ \\
Aerobias & 281 & $269(95,7)$ & $1(0,37)$ & $11(3,9)$ \\
Anaerobias estrictas & 280 & $242(86,4)$ & $1(0,41)$ & $37(13,2)$ \\
Total & 561 & $511(91)$ & $2(0,39)$ & $48(8,5)$ \\
\hline
\end{tabular}

Tabla 5. Discordancias obtenidas por identificación convencional vs secuenciación del gen 16 S ARNr en bacterias aeróbicas

\begin{tabular}{|c|c|c|c|}
\hline $\begin{array}{l}\text { Identificación } \\
\text { tradicional }\end{array}$ & $\begin{array}{l}\text { Identificación } \\
\text { MALDI TOF }\end{array}$ & $\begin{array}{c}\text { Puntaje } \\
\text { MALDI TOF }\end{array}$ & Identificación 16S ARNr \\
\hline Gemella spp & Rothia mucilaginosa & 2,167 & Rothia mucilaginosa \\
\hline Gemella spp & Granulicatella spp & 1,993 & Granulicatella paraadiacens \\
\hline Gemella morbilorum & Streptococcus cristatus & 2,177 & Streptococcus cristatus \\
\hline Gemella morbilorum & Streptococcus cristatus & 2,151 & Streptococcus cristatus \\
\hline Leuconostoc spp & Lactobacillus murinus & 2,06 & Lactobacillus murinus \\
\hline Micrococcus spp & Rothia mucilaginosa & 2,0 & Rothia mucilaginosa \\
\hline Micrococcus spp & Rothia mucilaginosa & 2,0 & Rothia mucilaginosa \\
\hline Micrococcus spp & Kokuria spp & 1,749 & Kokuria palustris \\
\hline Micrococcus spp & Kokuria spp & 1,98 & Kokuria rhizofila \\
\hline Micrococcus spp & Corynebacterium spp & 1,9 & Corynebacterium aurimucossum \\
\hline Capnocytophaga spp & Mycrobacterium spp & 1,987 & Mycrobacterium hydrocarbonoxydans \\
\hline Capnocytophaga spp & Streptococcus constellatus & 2,14 & Streptococcus constellatus \\
\hline Haemophilus aphrophilus & Actinobacillus urae & 2,271 & Actinobacillus sius \\
\hline Bordetella brochiseptica & Cupriavidus pauculus & 2,4 & Cupriavidus pauculus \\
\hline Haemophilus parainfluenza & Haemophilus parahemolyticus & 2,109 & Haemophilus parahemolyticus \\
\hline
\end{tabular}


Tabla 6. Detalle de los insumos y costos unitarios asociados a la identificación por MALDI TOF

\begin{tabular}{lcc} 
Insumos & $\begin{array}{c}\text { Costo sin extracción } \\
\$ \text { chilenos }\end{array}$ & $\begin{array}{c}\text { Costo con extracción } \\
\$ \text { chilenos }\end{array}$ \\
Guantes & - & 110 \\
Agua destilada & - & 2 \\
Etanol & - & 30 \\
Ac. tricloroacético & - & 1 \\
Ac. fórmico & - & 3 \\
Acetonitrilo & 60 & $\times 3$ u $=180$ \\
Puntas de $1 \mu \mathrm{l}$ & - & $\times 3 \mathrm{u}=270$ \\
Puntas de $100 \mu \mathrm{l}$ & - & $\times 2 \mathrm{u}=200$ \\
Puntas de $1.000 \mu \mathrm{l}$ & 80 & 80 \\
Control positivo & 200 & 200 \\
Matriz & - & 40 \\
Eppendorf $1,5 \mathrm{ml}$ & 2 & 2 \\
Eppendorf $0,2 \mathrm{ml}$ & 130 & 300 \\
Mano de obra & $\$ 472 *$ & $\$ 1.436 * *$ \\
\hline Total & & \\
\hline *Aproximadamente US: 1.0 ** Aproximadamente US: 3.0 & \\
\hline
\end{tabular}

Los insumos necesarios para la identificación por MALDI-TOF se detallan en la Tabla 6 junto a sus respectivos costos. En promedio la identificación tradicional en nuestro laboratorio es de US\$ 8 por cada muestra dependiendo del método utilizado lo que depende directamente de la familia a la que pertenezca dicha cepa. Sin embargo, el costo de identificación por MALDI-TOF MS es independiente de la familia y es, en promedio, de US\$1. Este costo varía dependiendo de si la cepa en estudio requiere de extracción previa o no.

Por último, el tiempo en la entrega de resultados desde que se obtiene crecimiento bacteriano hasta que el resultado es recibido por el médico clínico fue de seis horas cuando la identificación es por MALDI-TOF MS; el tiempo de entrega de resultados por los métodos tradicionales (considerando bacterias anaeróbicas estrictas) en promedio fue de $36 \mathrm{~h}$. Esta disminución en el tiempo de obtención de los resultados permite el informe de ellos dos veces al día.

\section{Discusión}

La identificación tradicional en el laboratorio de microbiología se basa en el uso de pruebas fenotípicas que normalmente requieren de largos períodos de incubación. La espectrometría de masas, si bien había sido utilizada en el campo de la microbiología básica, no se había adaptado para su uso en el laboratorio clínico microbiológico. Sin embargo, en el año 2008 dos publicaciones independientes reportan el uso de esta tecnología para la identificación de bacilos gramnegativos aislados de pacientes ${ }^{12,13}$. Desde entonces, numerosas publicaciones han demostrado su utilidad en el laboratorio clínico ${ }^{12-18}$; sin embargo, hasta nuestro conocimiento, no existen evaluaciones locales en América Latina de la utilidad de MALDI-TOF MS en la identificación de bacterias clínicamente relevantes.

La serie estudiada contempla 561 aislados de diversos géneros y especies, en los cuales, MALDI-TOF MS identificó adecuadamente a $95,7 \%$ de los aeróbicos y $86,4 \%$ de los anaeróbicos estrictos. Los porcentajes obtenidos para los aislados aeróbicos y anaeróbicos estrictos concuerdan con los publicados en la literatura científica en que los porcentajes de identificación correcta varían entre 93 y $100 \%{ }^{14,15}$.

El mayor porcentaje de identificación a nivel de especie se obtuvo en el grupo de Enterobacteriaceae. Es conocido que la identificación de Enterobacteriaceae por MALDI-TOF MS es cercana al $95 \%{ }^{14-20}$, con excepción de Shigella spp debido a que comparte un perfil proteico muy similar con E. coli impidiendo su correcta diferenciación, razón por la cual no fueron incluidas en este estudio. En el caso del género Salmonella, MALDI-TOF MS permite la diferenciación de serovars de Salmonella enterica subespecie enterica especialmente serovars Enteritidis, Typhimurium, Virchow, Infantis y Hadar ${ }^{21}$. Sin embargo, en nuestro trabajo sólo incluimos dos aislados, por lo que no podemos confirmar lo descrito por la literatura científica. Por otro lado, se han descrito dificultades en la identificación de Enterobacter cloacae complex ${ }^{22}$, un grupo de seis especies que están íntimamente relacionadas (E. cloacae, E. asburiae, E. hormaechi, E. kobei, E. ludwigii y E. nimipressuralis), por lo cual, en el presente trabajo, sólo llegamos a identificación de género.

MALDI-TOF MS ha demostrado mucha utilidad en bacilos gramnegativos no fermentadores ${ }^{12-20}$, donde los sistemas convencionales son poco exactos para las especies menos frecuentes. Esto fue reproducido en nuestro laboratorio en que $93 \%$ de los aislados fueron identificados correctamente a nivel de especie, alcanzando a 96,5\% cuando se considera identificación a nivel de género. En el caso de las identificaciones correctas de los tres aislados del grupo Acinetobacter baumannii, se debe tener en cuenta que tanto los métodos convencionales como la versión de la base de datos utilizada, puede incluir erróneamente en este grupo a Acinetobacter nosocomialis ${ }^{23}$. En la identificación de bacterias del grupo HACEK y otros fastidiosos las identificaciones correctas reportadas en la literatura científica superan el $94 \%{ }^{24}$, similar al $95 \%$ obtenido en este estudio.

En cuanto a la identificación de cocáceas grampositivas, $100 \%$ de la cocáceas habituales y $83 \%$ de las inhabituales fueron identificadas de manera correcta. 
Otras publicaciones en que se compara MALDI-TOF MS con los métodos tradicionales, éste presenta un excelente rendimiento, especialmente en Staphylococcus coagulasa negativa donde MALDI-TOF MS presenta 93\% de identificaciones correctas en relación al 75\% obtenido con sistemas automatizados como Phoenix ${ }^{\circledR}$ (Becton Dickinson) y Vitek-2® (bioMerieux) ${ }^{25}$. Resultados similares se han obtenido en Streptococcus $\operatorname{spp}^{14,15,17,19,26}$ con excepción de Streptococcus pneumoniae y Streptococcus parasanguinis del grupo mitis en los cuales el fabricante sugiere pruebas adicionales dada la incapacidad del equipo para diferenciar estas especies. Para el género Enterococcus spp, MALDI-TOF MS permite la identificación correcta de E. faecium, E. fecalis, E. gallinarum y E. flavus, que por métodos convencionales es muy inexacta ${ }^{16,17}$. Nuestros resultados con sólo cuatro aislados mostraron todos puntajes mayores de $2.000 \mathrm{y}$ $100 \%$ de identificaciones correctas. En el caso de cocáceas grampositivas inhabituales, MALDI-TOF MS identificó a nivel de especie a $61 \%$ de los aislados, a nivel de género a $22 \%$ y no se obtuvo identificación en el $17 \%$ restante. Si bien estos porcentajes son menores en comparación a otros grupos de bacterias, la identificación convencional generalmente es muy lenta y engorrosa. Otros estudios han demostrado su utilidad en la identificación de géneros como Abiotrophia spp Granulicatella spp, Globicatella spp, Vagococcus spp, Aerococcus spp y Gemella spp $^{3,17}$.

En anaerobios estrictos, MALDI-TOF MS identificó de manera correcta a $86,4 \%$ de los aislados evaluados, con $80 \%$ de identificación a nivel de especie. Es importante destacar que $55 \%$ de los aislados de Prevotella spp estudiados no fueron identificados. Esa situación, compartida con otros géneros como Fusobacterium spp y Peptostreptococcus spp se debe a una carencia de espectros en la librería lo que debiera resolverse en el tiempo. Es así como cuando los espectros de anaerobios están en la base de datos, la identificación por espectrometría de masas es mejor que la identificación convencional ${ }^{14,17,27}$.

Si comparamos los porcentajes de identificaciones incorrectas por MALDI-TOF MS e identificación convencional, con el método de referencia (secuenciación) se observa que la espectrometría de masas es un método más confiable que los utilizados actualmente en el laboratorio. De manera similar al comparar los costos, cada identificación cuesta en promedio ocho veces menos que la convencional.

En conclusión, nuestros resultados muestran que MALDI-TOF MS es un método confiable, de fácil uso y de menor costo que los utilizados hasta el momento en el laboratorio y que, además dada su rapidez, permite una disminución en el tiempo de respuesta de los resultados, lo que podría impactar en el cuidado oportuno del paciente.

\section{Resumen}

Introducción: La tecnología MALDI-TOF MS (Matrix Assisted Laser Desorption Ionization-Time of Flight Mass Spectrometry) incorporada recientemente en el laboratorio de microbiología ha demostrando ser un método rápido y preciso para la identificación bacteriana. Objetivo: Evaluar el desempeño de MALDI-TOF para la identificación de aislados clínicos, comparar los costos asociados y el tiempo en la entrega de resultados en un laboratorio de rutina. Material y Método: Se evaluaron un total de 561 aislados de pacientes (281 aeróbicos y 280 anaeróbicos estrictos) identificados previamente por métodos convencionales, los que fueron identificados por MALDI-TOF. Las discordancias fueron resueltas mediante secuenciación del 16S ARNr. Resultados: MALDI-TOF identificó adecuadamente a 95,7\% de los aislados aeróbicos y $86,4 \%$ de los anaeróbicos estrictos, observándose el mayor porcentajes de identificación a nivel de especie en los grupos de enterobacterias y Bacteroides spp (95 y $100 \%$ respectivamente). La tasa de error de MALDITOF y métodos convencionales vs secuenciación fue de 0,39 y $9,4 \%$, respectivamente. El costo asociado por identificación fue ocho veces menor que el de los métodos tradicionales con una demora promedio de seis horas en la entrega de resultados. Conclusión: MALDI-TOF mostró ser una tecnología simple, precisa y de menor costo que los métodos tradicionales.

\section{Referencias bibliográficas}

1.- La Scola B, Raoult D. Direct identification of bacteria in positive blood culture bottles by matrix-assisted laser desorption ionization time-of-flight mass spectrometry. PLoS one 2009; 4: e8041.

2.- Ferreira L, Sánchez-Juanes F, Muñoz Bellido J L, González-Buitrago J M. Rapid method for direct identification of bacteria in urine and blood culture sample by matrixassisted laser desorption ionization time-offlight mass spectrometry. Clin Microbiol Infect
2011; 17 (7): 1007-12.

3.- Van Veen S Q, Claas E C J, Kuijper E J. Highthroughput identification of bacteria and yeast by matrix-assisted laser desorption ionizationtime of flight mass spectrometry in conventional medical microbiology laboratories. J Clin Microbiol 2010; 48 (3): 900-7.

4.- Lotz A, Ferroni A, Beretti JL, Dauphin B, Carbonnelle E, Guet-Revillet E, et al. Rapid identification of mycobacterial whole cells in solid and liquid culture media by matrix-assisted laser desorption ionization-time of flight mass spectrometry. J Clin Microbiol 2010; 48 (12):
4481-6.

5.- García P, Allende F, Legarraga P, Huilcaman M, Solari S. Identificación bacteriana basada en el espectro de masas de proteínas: Una nueva mirada a la microbiología del siglo XXI. Rev Chilena Infectol 2012; 29 (3): 263-72.

6.- Vaneechoutte M, Claeys G, Steyaert S, De Baere T, Peleman R, Verschraegen G. Isolation of Moraxella canis from an ulcerated metastatic lymph node. L Clin Microbiol 2000; 38 (10): 3870-1.

7.- Wilck M B, Wu Y, Howe J G, Crouch J Y, Edberg S C. Endocarditis caused by culture 
negative organisms visible by Brown and Brenn staining: utility of PCR and DNA sequencing for diagnosis. J Clin Microbiol 2001; 39 (5): 2025-7.

8.- Poggi H, Guzmán A M, García P, Lagos M. "PCR universal o de amplio espectro": Un aporte a la detección de bacterias y hongos en la práctica clínica. Rev Med Chile 2009; 137 : 1122-5.

9.- $\quad$ http://blast.ncbi.nlm.nih.gov/Blast.cgi

10.- http://www.ncbi.nlm.nih.gov/genbank/

11.- Clinical and Laboratory Standards Institute (CLSI). Interpretive Criteria for Identification of Bacteria and Fungi by DNA Target Sequencing; Approved Guideline. CLSI MM18 A.

12.- Mellmann A, Cloud J, Maier T, Keckevoet U, Ramminger I, Iwen P, et al. Evaluation of matrix-assisted laser desorption ionizationtime-of-flight mass spectrometry in comparison to $16 \mathrm{~S}$ rRNA gene sequencing for species identification of non-fermenting bacteria. J Clin Microbiol 2008; 46: 1946-54

13.- Degand N, Carbonnelle E, Dauphin B, Beretti J L, Le Bourgeois M, Sermet-Gaudelus, et al. Matrix-assisted laser desorption ionization-time of flight mass spectrometry for identification of non-fermenting gram-negative bacilli isolated from cystic fibrosis patients. J Clin Microbiol 2008; 46: 3361-7.

14.- Seng P, Drancourt M, Gouriet F, La Scola B, Fournier P E, Rolain J M, et al. Ongoing revolution in bacteriology: Routine identification of bacteria by matrix-assisted laser desorption ionization time-of-flight mass spectrometry. Clin Infect Dis 2009; 49: 543-51.

15.- Ferreira L, Vega S, Sánchez-Juanes F, González M, Herrero A, Muñiz M C, et al. Identifying bacteria using a matrix-assisted laser desorption ionization time-of-flight
(MALDI-TOF) mass spectrometer. Comparison with routine methods used in clinical microbiology laboratories. Enferm Infecc Microbiol Clin 2010; 28: 492-7.

16.- Bizzini A, Durussel C, Bille J, Greub G, Prod'hom G. Performance of matrix-assisted laser desorption ionization-time of flight mass spectrometry for identification of bacterial strains routinely isolated in a clinical microbiology laboratory. J Clin Microbiol 2010; 48: 1549-54.

17.- Bessède E, Angla-Gre M, Delagarde $Y$, Sep Hieng S, Ménard A, Mégraud F. Matrixassisted laser-desorption/ionization biotyper: experience in the routine of a University hospital. Clin Microbiol Infect 2011; 17: 533-8.

18.- He Y, Li H, Lu X, Stratton C W, Tang Y W. Mass spectrometry biotyper system identifies enteric bacterial pathogens directly from colonies grown on selective stool culture media J Clin Microbiol 2010; 48: 3888-92.

19.- Benagli C, Rossi V, Dolina M, Tonolla M, Petrini O. Matrix-assisted laser desorption ionization-time of flight mass spectrometry for the identification of clinically relevant bacteria. PLoS One 2011; 6: e16424.

20.- Saffert R T, Cunningham S A, Ihde S M, Jobe K E, Mandrekar J, Patel R. Comparison of Bruker Biotyper matrix-assisted laser desorption ionization-time of flight mass spectrometer to BD Phoenix automated microbiology system for identification of gram-negative bacilli. J Clin Microbiol 2011; 49: 887-92.

21.- Dieckmann R, Malorny B. Rapid screening of epidemiologically important Salmonella enterica subsp. enterica serovars by whole-cell matrix-assisted laser desorption ionizationtime of flight mass spectrometry. Appl Environ
Microbiol 2011; 77: 4136-46.

22.- Hoffmann H, Stindl S, Ludwig W, Stumpf A, Mehlen A, Monget D, et al. Enterobacter hormaechei subsp. oharae subsp. nov., $E$. hormaechei subsp. hormaechei comb. nov., and E. hormaechei subsp. steigerwaltii subsp. nov., three new subspecies of clinical importance. J Clin Microbiol 2005; 43 (7): 3297-303.

23.- Espinal P, Seifert H, Dijkshoorn L, Vila J, Roca I. Rapid and accurate identification of genomic species from the Acinetobacter baumannii $(\mathrm{Ab})$ group by MALDI-TOF MS. Clin Microbiol Infect 2012; 18: 1097-103.

24.- Couturier M R, Mehinovic E, Croft A C, Fisher M A. Identification of HACEK clinical isolates by matrix-assisted laser desorption ionization-time of flight mass spectrometry. J Clin Microbiol 2011; 49: 1104-6.

25.- Dupont C, Sivadon-Tardy V, Bille E, Dauphin B, Beretti J L, Álvarez A S, et al. Identification of clinical coagulase-negative Staphylococci, isolated in microbiology laboratories, by matrix-assisted laser desorption/ ionization-time of flight mass spectrometry and two automated systems. Clin Microbiol Infect 2010; 16: 998-1004.

26.- Lartigue M F, Héry-Arnaud G, Haguenoer E, Domelier A S, Schmit P O, Van der MeeMarquet N, et al. Identification of Streptococcus agalactiae isolates from various phylogenetic lineages by matrix-assisted laser desorption ionization-time of flight mass spectrometry. J Clin Microbiol 2009; 47: 2284-7.

27.- Nagy E, Maier T, Urban E, Terhes G, Kostrzewa M. ESCMID Study Group on Antimicrobial Resistance in Anaerobic Bacteria. Species identification of clinical isolates of Bacteroides by matrix-assisted laser-desorption/ ionization time-of-flight mass spectrometry. Clin Microbiol Infect 2009; 15: 796-802. 